\title{
Recent Studies on Schistosoma intercalatum: Taxonomic Status, Puzzling Distribution and Transmission Foci Revisited
}

\section{Joseph Jourdane $/{ }^{+}$,Vaughan R Southgate*, Jean René Pagès, Patrick Durand** Louis Albert Tchuem Tchuenté***}

\begin{abstract}
Laboratoire de Biologie Animale (UMR CNRS 5555), Université, Av. de Villeneuve 66860, Perpignan Cédex, France *Department of Zoology, The Natural History Museum, London, UK **Centre d'Etudes sur le Polymorphisme des Microorganismes (UMR CNRS-IRD 9926), Centre de Recherche IRD, Montpellier Cédex, France ***Laboratoire de Biologie Générale, Faculté des Sciences, Université de Yaoundé I, Yaoundé, Cameroun
\end{abstract}

Schistosoma intercalatum, which causes human rectal schistosomiasis in Africa, still presents a great interest for its imprecise taxonomic status and its puzzling distribution in Africa. Two geographically isolated strains of S. intercalatum are recognized, the Lower Guinea strain and the Congo strain, which differ from each other in a number of morphological, biological and biochemical characteristics. Recent molecular data using RAPD markers indicate high divergence between the two strains, with values of Nei and Li's similarity indice allowing recognition of two genetically distinct taxa: experiments on pre-and post-isolating mechanisms are in progress in order to re-evaluate the taxonomic status of this polytypic species. With regard to its geographical distribution, S. intercalatum is characterized by the existence of two stable endemic areas (localized in Lower Guinea and North East of Democratic Republic of Congo) which correspond to the historical areas of species discovery, and the emergence during the last 15 years of new foci of the Lower Guinea strain outside previously known endemic areas. The absence of local adaptation of the Lower Guinea strain to its intermediate host, supported by experimental studies, may help to facilitate the spread of this strain. Nevertheless, the present restricted distribution of this species remains puzzling, because its potential snail hosts (bulinids) are widely distributed throughout much of Africa. Recent experimental and epidemiological studies suggest that interspecific sexual interactions between human schistosomes could have a role in limiting the distribution of $\mathrm{S}$. intercalatum: the competitive sexual processes acting among human schistosomes show that $\mathrm{S}$. haematobium and $\mathrm{S}$. mansoni are always competitively dominant over $\mathrm{S}$. intercalatum. These epidemiological observations lead the authors to distinguish three kinds of transmission foci for S. intercalatum.

Key words: Schistosoma intercalatum - Schistosoma mansoni - Schistosoma haematobium - taxonomic status sexual interactions - epidemiology - transmission foci

Schistosoma intercalatum, which causes human rectal schistosomiasis in Africa, still presents a great interest for its biological diversity and its puzzling distribution in Africa. Two geographically isolated strains of $S$. intercalatum are recognized, the Lower Guinea strain and the Congo strain, which differ from each other in a number of characteristics, including pre-patent periods in intermediate and definitive hosts, cercarial shedding patterns, isoenzymes, egg morphology and intermediate host

\footnotetext{
${ }^{+}$Corresponding author. Fax: +33-04-6866.2281 E-mail: jourdane@univ-perp.fr

Received 14 May 2001

Accepted 25 July 2001
}

specificity (Wright et al. 1972, Frandsen 1978, Bjorneboe \& Frandsen 1979). A post-isolating reproductive barrier between these two strains has been suggested, characterized by $\mathrm{F}_{2}$ hybrid inviability (Frandsen 1978).

\section{TAXONOMIC STATUS}

Recent molecular data using RAPD markers indicate high divergence between the two strains (Pagès et al. 2001). RAPD profiles show polymorphic bands between the two strains to be considered as diagnostic bands (only $22 \%$ of the RAPD fragments were shared between $S$. intercalatum Congo and $S$. intercalatum Lower Guinea). The mean values of Nei and Li's genetic distance between the two strains are 0.596 which compares with a figure of 0.546 between two distinct schistosome species, S. haematobium and S. mattheei. A principal component analysis showed a well de- 
fined separation between $S$. intercalatum Congo and S. intercalatum Lower Guinea. Experiments on pre- and post-isolating mechanisms are in progress in order to re-evaluate the taxonomic status of this polytypic species by splitting it into two distinct species

\section{PUZZLING DISTRIBUTION}

With regard to its geographical distribution, $S$. intercalatum is characterized by the existence of two stable endemic areas (localized in Lower Guinea and North east of Democratic Republic of Congo), which correspond to the historical areas of species discovery. Over the past 15 years, the Lower Guinea strain has apparently spread geographically; either within the historically endemic countries or into new countries (Equatorial Guinea, São Tomé island, Nigeria and Mali). Sporadic cases of S. intercalatum infections have been reported from Central African Republic, Chad, Angola, Burkina Fasso, Congo, Uganda and Senegal, but no active foci of transmission have so far been described in these countries. Even if the number of $S$. intercalatum transmission foci seems to be increasing, the present restricted distribution of this species is still puzzling because its potential snail hosts (bulinids of the africanus group for the Congo strain and bulinids of the forskalii group for the Lower Guinea strain) are very widely distributed throughout much of Africa and display, at least with regard to $B$. forskalii, a good compatibility with the parasite whatever, their geographical origin (no local adaptation of the Lower Guinea strain to its intermediate host in contrast to the Congo strain).

Maps of the distribution of human schistosomes in Africa suggest that the presence of $S$. haematobium or S. mansoni might prevent that of $S$. intercalatum, suggesting the existence of interspecific competition processes, a well understood ecological factor acting in the dynamics of free living organisms. A series of recent experimental and epidemiological studies are providing more and more evidence of the role of interspecific sexual interactions in limiting the distribution of $S$. intercalatum in Africa (Southgate 1978, Tchuem Tchuenté et al. 1996).

Hybridizations between S. intercalatum and S. haematobium - Experimental studies have clearly demonstrated that there are no reproductive isolating barriers between schistosomes species belonging to the same group. The existence of genetic exchanges between schistosomes has been shown in natural transmission foci where different species are sympatric. Within the $S$. haematobium species group, examples of hybridizations either in man or animals are well documented (Southgate \& Rollinson 1987).
With regard to the sexual interactions between $S$. intercalatum and $S$. haematobium, these take place during hybridization of the two species, which occurs easily whenever their distribution overlaps. Nevertheless, hybridization leads to a progressive dilution of $S$. intercalatum genome into that of $S$. haematobium due to the fact that the populations of the latter species are always in the majority and that $S$. haematobium male worms are better at pairing with females of either species. A recent epidemiological study carried out at Loum, Cameroon, has showed the total replacement of $S$. intercalatum by $S$. haematobium through unidirectionnal introgressive hybridization over a period of about 30 years (Tchuem Tchuenté et al. 1997)

Interactions between $S$. intercalatum and $S$. mansoni - Sexual interactions between $S$. haematobium and $S$. intercalatum can not totally explain the restricted distribution of $S$. intercalatum, as $S$. haematobium is absent in many regions of Africa, where $S$. intercalatum does not exist. Additional factors which may affect $S$. intercalatum distribution have been examined. The hypothesis deals with competitive exclusion between this species and another human species of schistosome, S. mansoni. Indeed, the geographical distribution of these two species suggests that the presence of one species might exclude that of the other. Experimental studies on the competitive processes between these two species both regarding immunological responses and mating behaviour have been carried out (Tchuem Tchuenté et al. 1996)

In order to test the cross-protection between the two species of schistosomes, immunization experiments were carried out. Immunizations with $S$. mansoni confer significant resistance to challenge with $S$. intercalatum. The degree of resistance observed varies according to the vaccinating infection $(36.8 \%$ after vaccination with $S$. mansoni female, $44.6 \%$ after vaccination with males and females of $S$. mansoni, 57\% after vaccination with $S$. mansoni males). On the contrary, vaccination with $S$. intercalatum does no protect against infection with S. mansoni. The results on cross-protection in mice between the two non closely related $S$. mansoni and $S$. intercalatum provide evidence of a significant level of resistance against $S$. intercalatum. In all experimental situations corresponding to the various immunization protocols, uni- or bisexual infection with $S$. mansoni always leads to a protection against infection with $S$. intercalatum. Interestingly, the cross-protection observed between S. mansoni and S. intercalatum is non-reciprocal.

The few works performed on cross mating between poorly related schistosome species have highlighted that interactions involve recognition, 
attraction and mating of the sexual partners. Schistosoma species display a unique feature amongst the Trematoda because they are dioecious and form pairs in their definitive hosts. Different types of pairs formed after simultaneous infections of the host by the two species of schistosomes were studied. A total of seven experiments which fall into three types were carried out: (1) simultaneous infection without choice of mate: infections with the same number of male cercarie of one species and female cercarie of the other species; (2) simultaneous infection with total mate choice: infections with the same number of cercarie of both sexes of both species; (3) simultaneous infections with limited choice of mate: for each experiment, one sex of the two epecies was absent and the number of cercarie of the single sex species present was lower in order to increase the possibility of choice.

Simultaneous infections performed show the absence of any physiological barriers preventing encounter and mating of both species within the vertebrate. However, whenever choice is possible, mating does not occur in a random way, but preferentially between partners of the same species resulting in a significant prevalence of homospecific pairs. Moreover, the experiments demonstrated that $S$. mansoni males, in the absence of $S$. mansoni females, are as competitive as $S$. intercalatum males at pairing with $S$. intercalatum females (Tchuem Tchuenté et al. 1993). With regard to our model, the results showed that when $S$. intercalatum and $S$. mansoni are found together in the same definitive host, there is a choice of mate. Nevertheless, this isolating factor by "harmonious mating" is not absolute, since heterospecific pairs can be performed. $S$. mansoni males are more successful at pairing with females than S. intercalatum males. It is of interest to note that the parthenogenetically-derived offspring from the heterospecific pairs are sterile.

If it is assumed that the mouse model has some relevance to the human situation, an epidemiological hypothesis could be extrapolated from the data. The occurrence in man of such competitive mating interactions between $S$. intercalatum and $S$. mansoni would significantly reduce the reproductive success of $S$. intercalatum in areas of sympatry, because: (1) the populations of S. mansoni will probably be in the majority since the cercarial production of $S$. mansoni per snail is up to ten times greater than that of $S$. intercalatum; (2) the immunological protection induced by $S$. mansoni against $S$. intercalatum will greatly reduce the adult population in man; (3) the sex ratios of schistosome adults are invariably biased towards males.

In sympatric situations where both $S$. mansoni and $S$. intercalatum are present, it is possible to estimate the proportion of female $S$. intercalatum which remain able to pair with their own males. We have drawn the trajectories of female $S$. intercalatum which retained homologous mating in relation to the mean number of $S$. mansoni per host. This proportion decreases drastically when the mean number of $S$. mansoni per host increases. Less than $20 \%$ of female $S$. intercalatum retained an homologous mating when the mean number of $S$. mansoni per host is more than ten. Approximately, the model suggests that the basic transmission rate could be reduced by a factor of five independently of a possible cross-protection between the two species. However it is noted that the aggregation of worms does not greatly influence this pattern. Even when the mean number of $S$. mansoni per host is low, the combined effects of biased sexratio towards male and the competitive superiority of $S$. mansoni yield to reduce dramatically the number of $S$. intercalatum pairs. In conclusion, the interactions between the two species in both immunity and mating show that $S$. mansoni is competitively dominant over $S$. intercalatum, which makes it very difficult for $S$. intercalatum to become established in areas where $S$. mansoni is present. Therefore, the very wide distribution of $S$. mansoni in Africa prevents $S$. intercalatum from spreading significantly.

\section{SCHISTOSOMA INTERCALATUM FOCI}

It has been demonstrated that $S$. haematobium and $S$. mansoni are always competitively dominant over $S$. intercalatum. Therefore, $S$. intercalatum will be at a disadvantage compared with either of the two species in situations of sympatry. Recent epidemiological data concerning $S$. intercalatum support our conclusion. Indeed, the foci described recently on the São Tomé and in Equatorial Guinea where transmission of $S$. intercalatum has now been established for several years, correspond to situations where both $S$. haematobium and $S$. mansoni are absent.

From these observations, three kinds of transmission foci for $S$. intercalatum can be distinguished.

The first is represented by the historical endemic foci in forest areas of Central Africa (Democratic Republic of Congo, Cameroon and Gabon) where $S$. intercalatum is the sole human schistosome present. In foci within the rain forest, both $S$. mansoni and S. haematobium can not perform their life cycle due to the absence of the appropriate intermediate hosts.

The second would include foci which have apparently emerged within the two past decades (São Tomé, Equatorial Guinea) where the parasite is able to establish on a long-term because neither $S$. haematobium or S. mansoni are present. 
The third would consist of mixed foci where the occurrence of $S$. mansoni or S. haematobium do not allow the definitive establishment of $S$. intercalatum. The presence of $S$. intercalatum in these foci could be occasional if there was only one introduction of the parasite (that could be the case of foci in Angola, Chad, Central African Republic Nigeria, Uganda where $S$. intercalatum seems to have been reported only once) or characterized by successive colonizations and extinctions in relation with a series of introductions of $S$. intercalatum by infected migrant populations (the focus in Dogon country, Mali, could correspond to this type).

\section{REFERENCES}

Bjorneboe A, Frandsen F 1979. A comparison of the characteristics of two strains of Schistosoma intercalatum Fisher, 1934 in mice. J Helminthol 53: 195-203.

Frandsen F 1978. Hybridization between different strains of Schistosoma intercalaum Fisher 1934 from Cameroon and Zaire. J Helminthol 52: 11-22.

Jourdane J, Southgate VR 1992. Genetic exchanges and sexual interactions between species of the genus Schistosoma. Res Rev Parasitol 52: 21-26.

Pagès JR, Durand P, Southgate VR, Tchuem Tchuenté LA Jourdane J 2001. Molecular arguments for splitting Schistosoma intercalatum, into two distinct species. Parasitol Res 87: 57-61.
Southgate VR 1978. On factors possibly restricting the distribution of Schistosomaintercalatum Fisher, 1934. Z Parasitenkunde 56: 183-193.

Southgate VR, Rollinson D 1987. The natural history of transmission and schistosome interactions. In D Rollinson, AJG Simpson (eds), The Biology of Schistosomes: from Genes to Latrines, Academic Press, p. 347-378.

Southgate VR, Jourdane J, Tchuem Tchuenté LA 1998. Recent studies on the reproductive biology of the schistosomes and their relevance to speciation in the Digenea. Int J Parasitol 28: 1159-1172.

Tchuem Tchuenté LA, Imbert-Establet D, Delay B, Jourdane J 1993. Choice of mate, a reproductive isolating mechanism between Schistosoma intercalatum and S. mansoni in mixed infections. Int J Parasitol 23: 179-185.

Tchuem Tchuenté LA, Morand S, Imbert-Establet D, Delay B, Jourdane J 1996. Competitive exclusion in human schistosomes: the restricted distribution of Schistosoma intercalatum. Parasitology 113: 129136.

Tchuem Tchuenté LA, Southgate VR, Njiokou F, Njiné T, Kouemeni LE, Jourdane J 1997. The evolution of schistosomiasis at Loum, Cameroon: replacement of Schistosoma intercalatum by S. haematobium through introgressive hybridization. Trans $R$ Soc Trop Med Hyg 91: 664-665.

Wright CA, Southgate VR, Knowles RJ 1972. What is Schistosoma intercalatum Fisher, 1934? Trans $R$ Soc Trop Med Hyg 66: 28-64. 